Horizons philosophiques

\title{
Remarque sur le problème du mal et l'amour chrétien
}

\section{Yvon Provençal}

Volume 2, numéro 1, automne 1991

De Buenos Aires à Québec

URI : https://id.erudit.org/iderudit/800884ar

DOI : https://doi.org/10.7202/800884ar

Aller au sommaire du numéro

\section{Éditeur(s)}

Collège Édouard-Montpetit

\section{ISSN}

1181-9227 (imprimé)

1920-2954 (numérique)

Découvrir la revue

Citer cet article

Provençal, Y. (1991). Remarque sur le problème du mal et l'amour chrétien. Horizons philosophiques, 2(1), 55-70. https://doi.org/10.7202/800884ar d'utilisation que vous pouvez consulter en ligne.

https://apropos.erudit.org/fr/usagers/politique-dutilisation/ 


\section{Remarque sur le problème du mal et l'amour chrétien}

Le problème du mal trouve des échos dans un lointain passé mais il est devenu, dans la modernité, un obstacle difficile à surmonter pour la religion et pour la morale. Certains penseurs y ont vu non seulement le problème le plus fondamental de toute la pensée humaine, mais en plus, un thème obsédant pour la sensibilité morale, qui fait craindre pour toutes leurs convictions.

Bien sûr, c'est un problème qui concerne d'abord les théologiens et les penseurs religieux. Là, on peut le voir comme l'objection tout à la fois la plus facile, la plus forte et la plus courante qu'on puisse élever contre l'existence de Dieu ${ }^{1}$. Ce problème met en cause la sainteté de Dieu, c'est-à-dire d'un Dieu supposément bon qui a pourtant créé un monde où la souffrance existe et qui a permis la damnation. Les auteurs modernes, beaucoup plus que les auteurs anciens, répugnent à admettre simplement la liberté souveraine ou le "bon plaisir" de l'Auteur de toutes choses. II faut donc soupçonner, si l'on veut croire, des raisons cachées, inaccessibles à l'entendement humain, ou du moins non communicables ou non objectives. Alors on parle de «mystère» et on invoque la fidélité, la confiance,

1. Voir Roger Verneaux, Problèmes et mystères du mal, Paris, éd. du Vieux Colombier, 1956, p. 8. 
l'espérance et la foi, et puis, l'amour de Dieu et pour Dieu, qui apparaît comme l'ultime argument assez fort pour résister à la tempête du doute.

Par ailleurs, le problème du mal n'intéresse pas que les théologiens. Albert Camus y a vu un vrai obstacle pour l'humanisme. L'existence de la cruauté en ce monde, particulièrement quand des enfants en sont victimes, apparaît comme un fait intolérable. Déjà Charles Renouvier exigeait une solution, car il ne pouvait se résoudre à croire à un monde d'injustice et de cruauté ${ }^{\text {. }}$.

On serait tenté d'éluder le problème : ne sommesnous pas là dans le domaine de la pure subjectivité. Tous ne sont pas également sensibles aux aspects même les plus atroces de la réalité. Et puis, qu'est-ce donc que le "mal»? Pouvons-nous lui donner une définition objective ou bien dépend-il de chaque culture, de chaque société et même de chaque individu? Certes, il serait difficile, sinon impossible, de définir le détail de ce qui ne peut se tolérer. Aussi identifierait-on plutôt le "mal» avec le fait général qu'il $y$ a de l'intolérable, sous une forme ou sous une autre, pour chaque instance subjective, qu'il $y$ a de la souffrance mauvaise, c'est-à-dire non agréée, non mise à profit de façon compréhensible. C'est sinon le mal en soi ou l'action en soi mauvaise qui existent objectivement, du moins le sentiment du mal, d'être victime, de l'intolérable, qui est incontestable et répandu universellement. Faut-il l'accepter avec fatalisme ou bien faut-il plutôt se révolter? Pouvonsnous à cet égard dépasser certaines idées reçues?

Le problème du mal ne concerne pas que les croyants, que les chrétiens qui sont aux prises avec le doute, mais tous ceux qui sont en rapport avec les croyants, qui doivent échanger avec eux, s'entendre pour vivre en société avec eux. Celui qui rejette l'existence de Dieu parce qu'il le rend responsable du mal peut-il s'accommoder facilement de vivre en compagnie de ceux qui

2. Charles Renouvier, Les derniers entretiens, Paris, A. Colin, 1904, p. 60-61. 
prétendent aimer et adorer ce “Dieu d'amour" qui n'est plus qu'une idole sanglante? Le consensus des incroyants et des croyants peut-il s'établir sur des bases suffisamment solides; peut-il y avoir des constitutions objectives? L'attitude normale du chrétien devant le mal est d'admettre son incompréhension et de croire qu'il sortira quelque chose de bon de la souffrance. Cependant, s'il admet l'enfer (ce qu'exige, semble-t-il, l'orthodoxie de la foi), il se trouve tenant d'une doctrine qui paraît scandaleuse et même ridicule pour la plupart des penseurs modernes. J. Stuart Mill voyait l'enfer comme une "effroyable idéalisation de la méchanceté humaine ${ }^{3}$ » et le considérait comme un mauvais exemple pour la morale, susceptible d'inspirer des comportements barbares ou cruels.

Et même sans aller jusqu'à considérer l'enfer des chrétiens ou leur Dieu-créateur, que nous faut-il penser du Dieu-juge, du Dieu-colère (qui ne sont pas confinés à l'Ancien Testament mais se manifestent aussi dans les Évangiles)? Ces questions constituent de redoutables pièges pour la foi religieuse, mais elles intéressent aussi l'incroyant, le penseur en général, dans la mesure où le christianisme se présente aujourd'hui comme une option moderne, qui peut rivaliser en "humanité" ou en valeur éthique avec toutes les philosophies qui ont vu le jour dans l'histoire de la pensée.

II y a, dans le problème du mal, plus qu'une critique religieuse : la critique d'une sensibilité et une remise en question fondamentale de tout l'univers et de toute l'histoire humaine. Si on invoque l'"amour" de Dieu ou pour Dieu, ou encore l'«amour" humain en général, il reste à savoir ce que ce mot "amour» signifie. L'amour chrétien ou l'humanisme moderne peuvent-ils encore nous satisfaire, peuvent-ils encore nous réconcilier vraiment avec l'intolérable?

3. John Stuart Mill, Essais sur la religion, trad. M.E. Cazellec, Paris, Alcan, 1884, p. 107. 


\section{Quelques moyens déjà pris pour enrayer la révolte.}

Que le problème du mal déborde le cadre de la pensée religieuse chrétienne est déjà évident quand on s'avise de la critique faite par Épicure (selon Lactance) contre l'existence de Dieu : ou bien Dieu veut supprimer le mal et ne le peut, ou il peut et ne le veut pas. Épicure conclut : Dieu est donc ou impuissant ou méchant (ou encore impuissant et méchant). À cette critique on peut d'ailleurs opposer la conception augustinienne d'une esthétique divine, selon laquelle le mal de chaque être particulier concourt au bien de l'ensemble de l'univers. Quelquefois on précise : comme les ombres d'un tableau sont nécessaires à l'harmonie. Certains théologiens ${ }^{4}$ d'aujourd'hui admettent cette conception comme valable pourvu qu'on laisse en quelque sorte à Dieu le droit à une esthétique supérieure, qui ne se compare pas avec les esthétiques humaines.

Cependant, tous ne peuvent admettre la beauté du tableau universel. Par exemple, au XVII ${ }^{\ominus}$ siècle, Pierre Bayle en fait la critique. II fait remarquer que, si un père, un roi, un administrateur, etc., se conduisaient comme nous voyons se conduire Dieu dans sa création, nous le blâmerions. Voici comment un théologien moderne répond à cette critique. Sertillanges juge que cette sorte de comparaison est "absurdement inadéquate au problème ${ }^{5}$ " et il invoque d'ailleurs saint Augustin, lequel condamne ceux qui, parlant de Dieu «ne comparent pas Dieu à Dieu, mais à eux-mêmes" (Cité de Dieu, XII, XVII). Toutefois, s'il est absurde de comparer la responsabilité de Dieu à celle de l'homme, il serait possible d'arriver à une conclusion allant dans le même sens que Bayle, mais plus fortement encore : on pourrait en effet juger Dieu plus responsable que

4. Par exemple, Roger Verneaux, Problèmes..., p. 62-63.

5. A.D. Sertillanges, Le problème du mal, t. 1, "L'histoire», Paris, Montaigne, 1948, p. 228. 
le père humain puisqu'il est supposément tout-puissant et omniscient. Et si c'est la bonté de Dieu qu'il est absurde de comparer à celle de l'homme, alors on pourrait reprendre les propos de J. Stuart Mill : "Si la bonté de Dieu n'est pas ce que nous appelons la bonté, je renonce à rien comprendre, et si je dois être damné pour cela, que je le sois ${ }^{6} . "$

Toujours en réponse à la conception de saint Augustin, qui est à tendance esthétique, pour tenter de justifier le mal, plusieurs penseurs ont préféré en quelque sorte s'inscrire dans l' "ombre" du tableau. Camus, dans L'Homme révolté, écrit : "Si le mal est nécessaire à la création divine, alors cette création est inacceptable." II se montre d'accord avec Ivan Karamazov, le personnage de Dostoïevski qui affirme que même si la vie immortelle lui était offerte, il la refuserait, eu égard aux victimes innocentes de ce monde terrestre.

Une caractéristique des auteurs modernes qui invoquent l'existence du mal pour rejeter la foi est leur hardiesse, voire leur insolence de rigueur, quand ils parlent du Dieu-créateur des chrétiens. Francis Jeanson rejette l'idée d'un péché originel et affirme que c'est plutôt à Dieu de se faire pardonner de l'avoir créé, lui, comme individu humain7. À la limite, on trouve une protestation comme celle de Nietzsche : "S'il y avait des dieux, comment supporterais-je de n'être pas un dieu; donc il n'y a pas de dieux ${ }^{8}$." Dans le style passionné qui est le sien, Nietzsche exprime la hantise de l'être condamné à rester en deçà de certaines limites imposées.

Le penseur moderne plutôt favorable à la foi chrétienne tente de prendre ce problème à revers. Paul Ricœur, par exemple, comprend "le mal par la liberté humaine». " affirme, de plus, que c'est librement qu'il pose la «liberté»

6. Cité par A.D. Sertillanges, ibid., p. 54.

7. Francis Jeanson, La Foi d'un incroyant, Paris, Seuil, 1963, p. 102.

8. Cité par Henri Arvon, L'Athéisme, Paris, PUF, 1967, p. 114. 
de cette façon : c'est en prenant sur soi le mal, donc ses limites, qu'on se libère à un plus haut niveau ${ }^{9}$. Cette conception philosophique pourrait d'ailleurs être illustrée par la vie et les propos de Simone Weil. Celle-ci a constaté que la souffrance n'ennoblit pas l'homme et qu'elle le rend semblable à une chose. Cette vérité lui est apparue concrètement dans l'expérience du travail et des camps de concentration. En revanche, elle affirme que seule la souffrance consentie peut arrêter le mal ${ }^{10}$. Cela suppose cependant un choix libre fondé sur une réflexion suffisamment profonde.

Mais peut-on s'en remettre à l'idée d'un "plan divin" dont la transcendance nous le rendrait incompréhensible? John Stuart Mill refuse cet argument : «Toute indication de plan dans le cosmos est une preuve contre l'omnipotence de l'Être qui a conçu le plan ${ }^{11}$.» En effet, pourquoi Dieu n'atteindrait-il pas directement la fin heureuse qu'il semble nous souhaiter? Pourquoi nous forcer à fonctionner d'abord dans ce monde et quel est le sens d'une telle épreuve? La réponse est peut-être encore dans cette conception de Paul Ricœur mentionnée ci-dessus. Le mal réside, selon lui, dans une "faiblesse constitutionnelle" inhérente à l'homme : sa faillibilité même ${ }^{12}$. Aussi, ce n'est pas tant un éventuel "plan divin» qui serait en défaut que les limites de l'humain et sa liberté. Pouvons-nous nous contenter cette fois de cette solution?

Voici quelques difficultés. Cicéron déjà, et puis Pierre Bayle, ont trouvé que le don du libre arbitre rendait indirectement la Providence responsable du mal et de toutes

9. Paul Ricœur, Finitude et culpabilité 1. L'homme faillible (Philosophie de la volonté), Paris, Aubier Montaigne, 1960, p. 14-15.

10. Voir Thomas J.J. Altizer, Toward a New Christianity, éd. par T.J.J. Altizer, Harcourt, Brace \& Worth Inc., 1967 ("Readings in the Death of God Theology").

11. John Stuart Mill, Essais..., p. 163

12. Paul Ricœur, Finitude..., p. 11 
ses conséquences ${ }^{13}$. Roger Verneaux, théologien moderne, admet cette conception. Dieu, en créant des êtres finis, corruptibles et sensibles, libres, rend possible le mal. Ainsi, écrit cet auteur, Dieu ne peut être exempté de toute responsabilité ${ }^{14}$. II est vrai cependant que Verneaux, qui est de tendance thomiste, ajoute que l'homme est seul et pleinement responsable de sa damnation : Dieu abandonne qui l'a abandonné, conformément à son attitude biblique Perditio tua ex te, Israel, Osée, XIII, 9) ${ }^{15}$.

Toutefois - et c'est là que Verneaux et Ricœur sont en quelque sorte à renvoyer dos à dos - le Dieu de Verneaux est-il un vrai "Dieu d'amour", qui serait responsable et pourtant impitoyable à damner l'homme, et le Dieu de Ricœur serait-il un vrai "Dieu de liberté», qui ne prendrait sur lui la responsabilité de la finitude humaine?

Écoutons Louis Lavelle qui nous propose une solution plus globale. La révolte contre la condition générale de l'homme serait évitable de la façon suivante. II est impossible, écrit-il, d'imaginer un monde où il n'y aurait que du bien, car le bien serait inconcevable si l'on n'avait pas l'expérience du mal. Aussi peut-on penser que "la masse de douleurs qui remplit l'histoire" a porté la conscience humaine «jusqu'au niveau spirituel où elle est parvenue ${ }^{16}$ ». Pourtant on peut voir encore une faille dans ce raisonnement. S'il est impossible d'imaginer un monde où il n'y aurait que du bien parce que le bien ne peut se concevoir sans l'expérience du mal, c'est aussi et surtout parce que Lavelle ne peut imaginer plus d'un monde et que toutes les expériences doivent être confinées dans ce monde unique. Cependant l'hypothèse d'une pluralité de mondes réels détruirait son raisonnement : pourquoi pas un monde

13. Voir A.D. Sertillanges, Le problème..., p. 228.

14. Roger Verneaux, Problèmes..., p. 78

15. Ibid., p. 105

16. Louis Lavelle, Le mal et la souffrance, Paris, Plon, 1940, p. 32 et p. 129. 
des expériences mauvaises, monde tenu à l'écart par l'Administrateur de tout, et pourquoi pas un monde sans piège où les êtres que Dieu aime pourraient vivre librement, sans contraintes? Les penseurs qui tiennent absolument à faire vivre leur foi existante de chrétiens sont-ils donc assujettis à des conceptions étroites par le fait même? L'hypothèse proposée ici possède une certaine continuité avec le propos de Jeanson, selon lequel il n'y a pas de Satan mais plutôt des «structures mauvaises" qui nous aliènent ${ }^{17}$. Elle est néanmoins plus radicale parce que Jeanson considère que le monde lui-même doit être accepté. II explique que c'est dans ce monde que nous pouvons progressivement surmonter nos diverses aliénations. Pourtant, même si cela est vrai pour certains, il est évident que tous n'en profitent pas également et qu'il y a là irrémédiablement des victimes qui doivent payer en quelque sorte pour les autres. Alors l'idée de ce monde unique, à laquelle Jeanson se confine comme les penseurs religieux, l'oblige encore à admettre avec complaisance - ou fatalisme? - l'existence des structures victimantes.

Revenons donc à Ricœur et à son idée de la faiblesse constitutionnelle de l'homme, qui rend possible ultimement le mal. Ce que cet auteur semble oublier est le mal que constitue - d'entrée de jeu - la possibilité même de ce qui apparaît comme un traquenard où l'individu et sa société, l'homme et son histoire sont piégés d'avance. Si l'on ne veut pas y voir un "piège» au sens propre, ce serait du moins un fatum à ne pas ériger en absolu, un destin à dépasser.

Cette conception, ici suggérée, pourrait par ailleurs faire penser à la "théologie de l'espérance» de J. Moltmann, selon laquelle ce n'est pas l'hybris le vrai mal mais plutôt le désespoir et la lâcheté ${ }^{18}$. En fait, ce qu'il faut peut-

17. Francis Jeanson, La Foi..., p. 151.

18. Voir Heinrich Fries, La Foi contestée (Herausgeforderter Glaube, 1968), trad. H. Rochais, Paris, Casterman, 1970, p. 100-108. 
être concéder ici au christianisme est une certaine valeur de ses propos sur le problème du mal. Les autres conceptions existantes ne vont pas plus loin. Le christianisme met par exemple de l'avant le "scandale de la croix", où un Dieu se fait tuer par des hommes, par amour pour eux. Ce scandale de la croix fait alors un peu contrepoids au scandale du mal. Quel Dieu, dans quelle autre religion, s'est jamais présenté ainsi : une incarnation au niveau de l'humain pour aider l'humain à surmonter le mal? Quelle philosophie peut opposer une idée plus forte que celle de l'amour infini pour tenter de rendre tolérable la condition générale de l'humain? Paul Valéry a écrit dans ses $\mathrm{Ca}$ hiers : "Le mot amour ne s'est trouvé associé au nom de Dieu que depuis le Christ." Encore faut-il savoir ce que l'on entend par "amour" et si l'on peut ou doit s'en satisfaire.

\section{L'amour chrétien est-il la meilleure réponse?}

Le christianisme est souvent défini comme une "religion d'amour", son Dieu comme un "Dieu d'amour". En effet, selon saint Jean : "Dieu est amour 19 " et il y a le précepte d'amour : "Tu aimeras ton prochain comme toimême ${ }^{20}$." Cet amour du prochain, ou amour de l'autre, est l'agapè, que tantôt on oppose à l'eros, amour du beau, tantôt à la philia, attirance du semblable pour le semblable. L'agapè, lui, ne fait pas de distinction et ne recherche aucune rétribution pour ses investissements ${ }^{21}$. Ce serait donc un amour désintéressé pour l'autre en général.

Considérons cependant la notion d'amour oblatif, qui est l'amour pour un autre être lui-même. L'accent est mis, au sens où on veut l'entendre ici, sur l'aspect vraiment désintéressé de l'amour oblatif. En ce sens, ce type

19. Première épître de Jean, iv, 8.

20. Matthieu, $22: 37$; Lévitique, $19: 18$.

21. Selon Paul van Buren. Voir Thomas J.J. Altizer, Toward..., p. 287. 
d'amour ne recherche de rétribution que dans la satisfaction inhérente au respect de l'autre en tant qu'autre et dans la satisfaction de savoir que l'autre se réalise selon les visées qui sont les siennes. II n'y a donc là aucune rétribution sous la forme d'un manque assouvi vis-à-vis de l'autre, sous la forme d'un attachement de l'autre à sa personne ou sous la forme d'une réalisation de l'autre selon des vues conformes à une soi-disant universalité dont on l'investit contrairement au désir de réalisation qui est celui de l'autre.

II est clair que l'amour qui consiste à remercier des bienfaits pour en mériter d'autres ou qui consiste à se soumettre à de mystérieux desseins d'un Pouvoir supérieur n'est pas un amour oblatif. C'est plutôt, selon Francis Jeanson, l'«étrange amour" qu'on peut trouver dans l'Ancien Testament ${ }^{22}$. De plus, l'amour du Dieu créateur peut s'attirer plusieurs critiques. Notons d'abord que l'on peut considérer la création comme «un don de l'existence ${ }^{23}$ » et qu'elle est le fait de «la générosité créatrice ${ }^{24} »$. Or la nature ne fonctionne pas sur le principe de l'amour mais plutôt sur celui de la prédation. John Stuart Mill trouve, pour sa part, que la nature est "plus cruelle que les hommes». Elle fait preuve, selon lui, d'une ingénieuse cruauté que même un Nabis ou un Domitien n'ont jamais surpassée 25 . De plus, elle est foncièrement injuste parce que c'est celui qui est déjà le mieux doté qui accapare toujours plus. Par exemple, le riche gagne de l'argent sans peine ${ }^{26}$. Pour Pierre Bayle, trois siècles avant Stuart Mill, la nature est

22. Francis Jeanson, La Foi..., p. 123.

23. R. Verneaux, Problèmes..., p. 89.

24. A.D. Sertillanges, Le problème..., p. 294.

25. John Stuart Mill, Essais..., p. 27

26. Ibid., p. 33. On notera que John Stuart Mill semble considérer que la répartition des richesses est un résultat “naturel», mais ce qui importe ici est la connotation réprobatrice qui accompagne ses propos. 
un état de maladie où tout passe et tout se corrompt. Schopenhauer, après Hume, va plus loin et juge que ce monde est le pire des mondes possibles. Tout y est soumis au risque des perturbations et des accidents. En somme, ces auteurs ne font que prendre acte de l'existence d'un mal inhérent à la réalité naturelle - sous la forme des accidents, de la maladie et de la mort - et ils le font dans l'idée d'une critique à l'encontre de la bonté divine ou de son omnipotence. Et si ce n'était là que le résultat du péché libre de l'homme, il resterait à prouver, contre toute vraisemblance, que l'inférence du péché à la cruauté des choses peut être établie de façon compatible avec l'idée d'un «Dieu d'amour».

Il est vrai par ailleurs que la foi chrétienne, particulièrement quand elle se nourrit des Évangiles et des propos du Christ, incite à l'amour des êtres humains plus que la majorité des philosophies ou des autres religions, sinon plus que toutes les philosophies et toutes les autres religions. Par exemple, Jésus, contrairement à Platon ou Aristote, semble avoir une conception illimitée de l'autre humain digne de respect ou d'amour27. II dit même : “Aimez vos ennemis,..." (Matthieu, V, 44). On a souvent opposé la loi d'amour de Jésus à l'amour de la loi, ce que certains ont interprété dans le sens d'une opposition du Nouveau à l'Ancien Testament, de Jésus à Yaveh ou le Roi-gendarme. Le fait est que les enseignements de Jésus prennent souvent plus d'importance dans la morale chrétienne que ceux de la Bible auxquels on accorde surtout une importance historique et parfois pédagogique ${ }^{28}$.

Aussi ne faut-il pas s'étonner d'arguments en faveur de la foi chrétienne comme celui du père Valensin, dans

27. Voir, par exemple, Luc, $10: 29-37$. Le Samaritain, étranger et hérétique, est opposé à un lévite, qui compte parmi ce qu'il y a de plus respectable en Israël.

28. Le précepte de l'amour chrétien est formulé dans le Pentateuque, mais le Christ lui a donné un sens plus fort. 
sa "prière impossible»: si je devais perdre la foi, "c'est l'amour infini qui aurait tort de ne pas exister et non pas moi d'avoir cru en lui»; ou encore comme celui du curé d'Ars : «Je serai bien attrapé" si ce n'est pas vrai, mais je serai content d'avoir cru en l'amour ${ }^{29}$. Ce type d'argument peut s'appuyer à juste titre sur certains traits uniques de la doctrine chrétienne.

Toutefois plusieurs auteurs ont fait remarquer que les propos et les attitudes du Christ n'étaient pas toujours exempts de dureté, voire de cruauté. Ainsi, on a relevé ce trait de chauvinisme de Jésus quand il traite les Cananéens, ou habitants de Tyr et Sidon, de "petits chiens" par opposition aux "enfants d'Israë| 30 ". II refuse même d'abord d'aider une Cananéenne qui l'implore, en raison de ses origines. Certes, Jésus était un Juif et il se comportait en accord avec les mœurs de l'époque. L'argument de son humanité nous le rend en un sens plus proche, mais il a également pour effet de banaliser certaines de ses attitudes, de leur enlever une part de leur caractère exemplaire dans son rôle de "Dieu d'amour". D'autant plus que le Christ a souvent tenu des propos violents contre certains individus, stigmatisés alors pour leur état ou classe sociale (pharisien, scribe) ou bien pour leurs actes. Un exemple est ce long passage des imprécations contre les "scribes et pharisiens hypocrites ${ }^{31}$ ". Un autre exemple est le passage redoutable où il parle d'attacher une meule au cou de qui scandalise un de ces petits qui croient en lui, et de le précipiter au fond de la mer ${ }^{32}$. Là, l'amour du Christ ne se montre pas meilleur que celui des Psaumes avec leur vœu obstiné de l'extinction des méchants. On pourrait objecter ici que l'amour est conciliable avec la

29. Cité par Jean Guitton, Ce que je crois, Paris, Grasset, 1971, p. 163-164.

30. Matthieu, $15: 21-28$ et Marc, $7: 24-30$.

31. Matthieu, $15: 3-20$.

32. Matthieu, $18: 6$; Marc, $9: 42$. 
colère, et peut-être même que celle-ci souligne celui-là. Toutefois, cette conception de l'amour-colère n'est guère compatible avec celle de l'amour oblatif où l'autre est considéré profondément respectable dans sa liberté et ses options propres. Qu'une certaine cruauté soit de mise dans un monde de violence est possible, mais cela n'a pas à être confondu avec l'amour. Le Christ visiblement cède à cette cruauté.

Cette confusion entre l'amour authentiquement oblatif et une certaine violence à l'égard de l'autre en tant qu'autre se produit fréquemment. On cite dans un but d'édification cette phrase de saint Jean de la Croix : "Nous serons jugés sur l'Amour." On ne se rend pas compte que cette proposition comporte des éléments contradictoires. En effet, "nous serons jugés" implique quelque chose comme un souci de justice, d'harmonie contrainte, mais cela va à l'encontre de l'amour oblatif. Dieu peut bien juger ses créatures, ce n'est pas en cela du moins qu'il est un Dieu d'amour. Le «jugement» au sens chrétien est une opération qui viole la liberté de l'autre en tant qu'autre.

N'y a-t-il pas néanmoins, dans les aspects les plus essentiels de la doctrine chrétienne une notion d'amour agapè vraiment désintéressé? Par exemple, Louis de Naurois définit l'amour comme étant "communion entre personnes, don et accueil mutuel ${ }^{33}$ ". On voit alors Jésus comme l'exemple et le critère d'un amour qui rencontre et qui se donne. Certes, l'amour oblatif n'exclut pas la rencontre et le don vis-à-vis de l'autre. II ne les force cependant pas. Là semble être toute la différence - et elle est essentielle - entre l'amour oblatif comme tel et l'amour chrétien. Le premier respecte le désir de l'autre même si ce désir donne lieu éventuellement à un refus de l'échange au niveau inter-subjectif d'une communauté unique. II respecte l'autre qui choisit un autre monde, d'autres règles ou

33. Louis de Naurois, Problèmes éthiques de l'avortement. Documents Santé mentale, Toulouse, Privat, 1973, p. 120. 
même l'absence de règles. II ne le condamne ni ne le damne pour autant ${ }^{34}$.

Ceci nous amène à éclaircir certaines situations confuses. Le penseur chrétien, même quand il se permet certaines libertés de jugement vis-à-vis de la doctrine officielle, continue en général de croire en la damnation (voire en l'enfer). Il est très intéressant de voir comment on tente d'accorder l'idée de la damnation avec celle de l'amour infini. Roger Verneaux écrit que Dieu laisse souffrir le damné parce que cela est conforme à la volonté du damné et par souci de justice ${ }^{35}$. II y a là une confusion manifeste qui réside dans l'expression "volonté du damné» : celui-ci a voulu sans doute déroger aux règles édictées par le ciel, peut-être veut-il aussi sa propre solitude; il ne veut pas pour autant être puni et souffrir éternellement. Quant au "souci de justice", il ne peut résulter de l'amour oblatif comme tel, car il impose à l'individu une exigence d'appartenance à une communauté qu'il rejette.

D'ailleurs, on accepte généralement que soit condamné l'individu qui tente d'imiter Dieu, ou qui prétend dépasser son statut humain, qui se rend coupable de l'orgueil sacrilège. Pourtant, quand un père aime son enfant, il ne le condamne pas de viser à lui ressembler, voire d'aspirer à le dépasser : au contraire. Il est vrai qu'il s'agit ici d'un père humain. Dieu serait-il donc moins capable d'un amour oblatif véritable que l'être humain?

Précisément, un Être dont la volonté n'a pas d'autre fin qu'elle-même - ce qui revient à la définition même de Dieu - peut-il aimer l'autre en tant qu'autre? On notera ici que le caractère absolu de l'Être n'a pas pour effet de

34. Dans Des choses cachées depuis la fondation du monde (Paris, Grasset, 1978), René Girard voit l'amour chrétien comme l'ultime solution pour éviter de sombrer dans le nihilisme. II fait alors de l'amour une sorte d'instrument collectif de survie. II vise surtout les rapports entre les diverses sociétés. Cette conception en quelque sorte utilitaire de l'amour ne peut être considérée comme une forme d'amour oblatif.

35. Roger Verneaux, Problèmes... p. 201. 
le rendre non aimable : toute l'histoire du christianisme le démontre. Cependant, ce caractère absolu semble le récuser pour l'amour oblatif en tant que tel. Aussi bien, la notion de Dieu en tant qu'absolu a fait dire à des auteurs de bonne foi que Dieu n'a pas pour fonction de résoudre les problèmes de l'homme ${ }^{36}$ ou que Dieu élit qui il veut, aime plus ou moins selon sa souveraine libertés7. Ces propos semblent démontrer une chose : les êtres humains (ces auteurs en particulier) sont plus capables d'amour oblatif à l'égard d'un être autre - à savoir Dieu - que celui-ci ne l'est à l'égard des êtres humains. Aussi pourrait-on conclure que le Dieu que les chrétiens ont conçu n'est pas à la hauteur des chrétiens eux-mêmes et, partant, des humains en général.

Francis Jeanson a écrit : Aimer quelqu'un quoi qu'il fasse, c'est le nier délibérément ${ }^{38}$. L'incroyance même de cet auteur montre qu'il aurait été d'accord pour viser ainsi l'amour du croyant pour Dieu. En tout cas, lui, Jeanson, s'est vu contraint de nier Dieu. Ceci dit, l'amour oblatif n'oblige pas à nier l'autre. C'est au contraire celui qui n'éprouve pas ce type d'amour pour l'autre qui peut nier l'autre en tant qu'autre. Pour cela, il faut cependant considérer des possibilités nouvelles pour la réalité admissible : l'univers étroit dans lequel on est confiné, et dont l'unicité absolue est inhérente à la finitude mauvaise de l'humain, doit pouvoir être dépassé, à défaut de quoi l'amour oblatif n'aura que peu d'objet. Accepter l'autre en tant qu'autre suppose en effet la possibilité pour l'autre de se réaliser ailleurs. Dépasser cet univers-ci - ou plutôt son caractère absolu et unique - ne signifie d'ailleurs pas se nier soimême, à moins de présupposer de façon restrictive les

36. T.W. Ogletree, La controverse sur la "mort de Dieu» (The Death of God Controversy, 1966), trad. J. Cloarec, Tournai, Casterman, 1968, p. 38.

37. R. Verneaux, Problèmes..., p. 107.

38. Francis Jeanson, La Foi..., p. 132. 
limites de soi-même, ce qui n'est peut-être alors qu'une résurgence du vieux fatalisme religieux. Ces contraintes abusives qu'on pose à soi-même, voilà sans doute ce qui rend compte de l'efficacité relativement faible, tout au long de l'histoire, du précepte chrétien : "Aime ton prochain comme toi-même." Aussi bien, aimer l'autre "comme soimême" est peut-être très mal aimer l'autre, particulièrement quand le soi se limite trop. Pourquoi pas plutôt : "Aime l'autre comme il désire être aimé et dans la mesure même où il désire être aimé". Cette formulation serait beaucoup mieux en accord avec la conception oblative de l'amour. Elle engagerait sans doute à plus d'imagination et puis, elle inciterait davantage à sortir du "petit cachot ${ }^{39}$ ".

YVON PROVENÇAL Collège de Granby

39. Le "petit cachot» est - rappelons-le - l'expression utilisée par Teilhard de Chardin pour désigner l'étroitesse de l'univers existant. 